\title{
Perfil de resistencia de la Salmonella sp durante un periodo de tres años en un hospital de Colombia
}

\author{
Resistance Profile of Salmonella SP During \\ a Period of Three Years in a Hospital of Colombia \\ Perfil de resistência de Salmonella SP \\ durante um período de três anos em \\ um hospital da Colômbia \\ María Alejandra Pérez Ardila, $\mathrm{MD}^{1 *}$ \\ Iván Noreña, $\mathrm{MD}^{2}$ \\ Henry Augusto Millán, MD² \\ Julián Alberto Naranjo, MD \\ Juan Castellanos, MD' \\ Laura Ladino, $\mathrm{MD}^{1}$ \\ Fredy Andrés Luna, MD ${ }^{1}$ \\ Laura Fernanda Rincón, MD $^{3}$ \\ Luisa Duarte, Bact ${ }^{4}$
}

Recibido: 4 de junio de 2019 - Aceptado: 25 de octubre de 2019

Doi: https://doi.org/10.12804/revistas.urosario.edu.co/revsalud/a.8768

Para citar este artículo: Pérez M, Noreña I, Millán H, Naranjo J, Castellanos L, Luna F, Rincón L, Duarte L. Perfil de resistencia de la Salmonella sp durante un periodo de tres años en un hospital de Colombia. Rev Cienc Salud. 2020;18(1):108-118. https://doi. org//10.12804/revistas.urosario.edu.co/revsalud/a.8768

\section{Resumen}

Introducción: en Colombia, del 2000 al 2012, se describió un aumento progresivo en la resistencia a betalactámicos y quinolonas en los aislamientos de Salmonella sp. A partir de esta fecha se desconoce la evolución de las tasas de resistencia en el país. El objetivo de este estudio fue describir el perfil de suscep-

1 Departamento de Infectología, Fundación Cardioinfantil, Bogotá, Colombia.

Autor de correspondencia: mariaperezardila@gmail.com

2 Departamento de Infectología, Fundación Cardioinfantil, Bogotá, Colombia.

3 Universidad Industrial de Santander. Bucaramanga, Colombia.

4 Departamento de Microbiología, Fundación Cardioinfantil, Bogotá, Colombia. 
tibilidad durante el periodo 2014-2017 así como evaluar la asociación entre las características clínicas y sociodemográficas de la población con los patrones de resistencia de Salmonella sp. Materiales y métodos: estudio de corte transversal del 2014 al 2017, realizado en la Fundación Cardioinfantil en mayores de 18 años, con aislamiento de Salmonella sp en cualquier tipo de muestra biológica. Resultados: se encontraron sesenta casos, ningún aislamiento fue resistente a quinolonas, uno mostró resistencia a ampicilina y uno se caracterizó como Amp-c; el 95 \% tenía perfil de susceptibilidad usual. No se encontró asociación entre las variables estudiadas y la presencia de resistencia. Conclusión: los resultados pueden reflejar un cambio en el perfil de susceptibilidad de Salmonella sp en Colombia, con una disminución en la resistencia a betalactámicos y quinolonas a partir del 2014. Sin embargo, se requiere una mayor muestra poblacional para corroborar esta hipótesis.

Palabras clave: Salmonella; infecciones por Salmonella; fiebre tifoidea; farmacorresistencia microbiana; Salmonella typhi; epidemiología.

\section{Abstract}

Objective: In Colombia, from 2000 to 2012, a progressive increase in resistance to beta-lactams and quinolones was described in isolates of Salmonella $S$. As of this date, the evolution of resistance rates in the country is unknown. The objective of this study is to describe the susceptibility profile from 2014 to 2017, as well as to evaluate the association between the clinical and sociodemographic characteristics of the population with the resistance patterns of Salmonella SP. Materials and methods: The study is a cross-sectional study between 2014 and 2017 at the Fundación Cardioinfantil, in patients over 18 years of age with Salmonella sP, in any type of biologic sample. Results: The authors found 60 cases, no isolate was resistant to quinolones. One showed resistant to ampicillin, and another one was characterized as amp-C; $95 \%$ had a usual susceptibility profile. No association was found between the variables studied and the presence of resistance. Conclusion: The results may reflect a change in the susceptibility profile of Salmonella SP in Colombia, with a decrease in resistance to beta-lactams and quinolones as of 2014; however, a larger population sample is required to corroborate this hypothesis.

Keywords: Salmonella; Salmonella infections; typhoid fever; microbial drug resistance; Salmonella typhi; epidemiology.

\section{Resumo}

Objetivo: Na Colômbia do ano 2000 ao 2012 se descreveu um aumento progressivo na resistência a beta-lactâmicos e quinolonas nos isolamentos de Salmonella sp. A partir desta data se desconhece a evolução das taxas de resistência no país. O objetivo deste estudo é descrever o perfil de susceptibilidade do ano 2014 ao 2017, assim como avaliar a associação entre as características clínicas e sociodemográficas da população com os patrões de resistência Salmonella sp. Materiais e métodos: Estudo de corte transversal do ano 2014 ao ano 2017 realizado na Fundación Cardioinfantil em maiores de 18 anos com isolamento de Salmonella sp, em qualquer tipo de amostra biológica. Resultados: se encontraram 60 casos, nenhum isolamento foi resistente a quinolonas, um mostrou resistência a ampicilina e um se caracterizou como AMP-C, o 95\% tinha perfil de susceptibilidade usual. Não se encontrou associação entre as variáveis estudadas e a presença de resistência. Conclusão: Os resultados podem refletir uma mudança no perfil de susceptibilidade de Salmonella $s p$. Na Colômbia, com uma diminuição na resistência a beta-lactâmicos e quinolonas a partir do ano 2014, no entanto se requere uma maior amostra populacional para corroborar esta hipótese.

Palavras-chave: Salmonella; infeções por Salmonella; febre tifoide; fármaco-resistência microbiana; Salmonella typhi; Epidemiologia. 


\section{Introducción}

$E^{1}$ género Salmonella forma parte de la familia Enterobacteriaceae y se divide en dos especies: Salmonella enterica y Salmonella bongori; la primera de ellas contiene los serotipos patógenos para los humanos. Se trata de bacilos Gram negativos anaerobios facultativos, no formadores de esporas, los cuales también fermentan glucosa, reducen nitrato y no producen citocromo oxidasa. El único serotipo que no produce gas en la fermentación de azúcar es S. typhi. Los serotipos S. typhi y S. parathyphi se conocen como los serotipos de Salmonella tifoidea, los demás se conocen como serotipos no tifoideos (1).

La mayor incidencia de infección por Salmonella se encuentra en pacientes con infección por virus de inmunodeficiencia humana ( $\mathrm{viH})$, enfermedad de células falciformes, niños y quienes viven en zona rural (2). Los serotipos más frecuentes son S. typhimurium (40\%), S. enteritidis (12\%) y S. dublin (11\%) (3)multicountry data are required to better understand the nature and burden of disease in Africa. We aimed to measure the adjusted incidence estimates of typhoid fever and invasive non-typhoidal salmonella (iNTS).

En el 2000, la fiebre tifoidea tenía una incidencia de 21 millones de casos y 217000 muertes cada año en el mundo (4). En el ámbito nacional, para el 2017, según el boletín epidemiológico del Instituto Nacional de Salud de Colombia la incidencia fue de 0.2 casos por 100000 habitantes y se notificaron 143 casos. El mayor porcentaje de casos confirmados correspondió a Antioquia, Bogotá, Bolívar, Meta y Norte de Santander. En ese año, de los casos confirmados por laboratorio el $66.3 \%$ eran de sexo masculino y $70 \%$ ocurrieron en cabecera municipal. El mayor porcentaje ocurrió en el grupo de 10 a 14 años, seguido del de 5 a 9 años (5).

El tratamiento hasta principios de 1990 estaba constituido por Cloranfenicol, ampicilina y cotrimoxazole, con el aumento en la incidencia de cepas resistentes el tratamiento de primera línea actual para las infecciones por Salmonella sp está constituido por fluoroquinolonas, azitromicina y cefalosporinas de tercera generación (6). Las tasas de resistencia en la última década varían entre los países, aumento de la resistencia en cepas en países como España, Brasil y China; y menores tasas de resistencia en Estados Unidos (7-10), siendo en este país la resistencia única a ampicilina el patrón más común en $6.5 \%$ de los aislamientos, resistencia en ceftriaxona y ampicilina en $3.7 \%$ y a ciprofloxacina en $0.2 \%$.

Dentro de los países mencionados con aumento en cepas resistentes es de importancia resaltar su variabilidad epidemiológica. En España, por ejemplo, la resistencia a ampicilina alcanza el $55 \%$ entre los aislamientos de Salmonella sp, con resistencia a ácido nalidíxico de $19 \%$, sin haber encontrando casos de resistencia a Ciprofloxacina (7). Mientras en Brasil la resistencia al ácido nalidíxico viene en ascenso desde el año 1999 cuando se reportaba del $12.8 \%$ hasta $89 \%$ en el año 2012 (8). En China, la resistencia a fluoroquinolonas también en ascenso reportó una frecuencia del 5.9\% para el año 2012 (9). 
En los reportes del Instituto Nacional de Salud del 2000 al 2012 en Colombia se observó un aumento progresivo en la resistencia al ácido nalidíxico, a la ampicilina, al cefotaxime y a la ciprofloxacina. El serotipo más aislado en el periodo 2000-2012 fue S. typhimurium, en el $33.7 \%$ de las muestras, seguido de $S$. enteritidis en $28.6 \%$ y $S$. typhi en $9.2 \%$ (11). En $S$. typhimurium, para el último período (2012), la resistencia fue de $16.6 \%$ al ácido nalidíxico, $2.6 \%$ a la ciprofloxacina, $15.8 \%$ a la ampicilina y $1.6 \%$ a la ceftriaxona. Sin embargo, a partir de la fecha, se desconoce el perfil de resistencia de Salmonella sp en el país.

Según lo anterior, el objetivo primario del estudio fue caracterizar el perfil de susceptibilidad de Salmonella sp en muestras biológicas obtenidas desde el 2014 hasta el 2017 en la Fundación Cardioinfantil-IC. Asímismo, explorar la asociación entre las características clínicas y sociodemográficas de la población con los patrones de resistencia de Salmonella sp.

\section{Materiales y métodos}

E studio transversal descriptivo a partir de registros de laboratorio que incluyó pacienEtes mayores de 18 años con aislamiento de Salmonella sp en urocultivo, hemocultivos, coprocultivo o cultivo de secreción provenientes de cualquier fluido (sangre, orina, heces o material purulento) de pacientes hospitalizados durante el período 2014 a 2017 en la Fundación Cardioinfantil- Ic (Hospital de carácter privado).

La información de aislamientos de Salmonella sp se extrajo a partir de la base de datos Whonet®, del laboratorio clínico de la Fundación Cardioinfantil-Ic, con el correspondiente perfil de susceptibilidad y el tipo de muestra de cada uno. No se calculó el tamaño de muestra dado que se tomó la totalidad de la población.

Los criterios de inclusión fueron: pacientes con aislamiento de Salmonella sp en cualquier material biológico que contaran con el perfil de susceptibilidad disponible y que se tratara de pacientes mayores a 18 años. Se excluyeron mujeres embarazadas. Este estudio fue aprobado por el comité de ética de la Fundación cardioinfantil-ic mediante el acta Nº 11-2018.

La siembra de las muestras con sospecha de infección por Salmonella sp se realizó en agares selectivos como Salmonella/Shigella y xLD (XyloseLysineDeoxycholate) y se realizó, en caso de ser necesario, aislamiento en Agar McConkey + Cristal Violeta (BioMérieux®).

Identificación y susceptibilidad microbiológica: se realizó identificación y susceptibilidad por el sistema Vitek 2xL (BioMérieux ${ }^{\circledR}$ ) empleando tarjetas para microorganismos Gram negativos GN y AST-272, respectivamente. La sensibilidad fue determinada de acuerdo a los estándares del Clinical and LaboratoryStandardsInstitute (cLSI) (12).

Datos sociodemográficos y clínicos: se empleó el sistema de información ServinteClinical Suite Enterprise ${ }^{\circledR}$ para extraer datos sociodemográficos y clínicos al momento de la infección. 
Las variables cuantitativas de estudio se calcularon medidas de tendencia central. En las variables con una distribución normal se realizó el cálculo de medias y desviación estándar, de otra forma se describen mediante medianas y rangos intercuartílicos. Las variables cualitativas se resumieron por medio de frecuencias relativas y absolutas.

Se tomaron las siguientes definiciones para los patrones de descripción fenotípica:

- Patrón de resistencia usual: Salmonella sp presenta resistencia a cefalosporinas de primera y segunda generación, así como a cefamicinas y aminoglucosidos (12).

- BLEE (Beta lactamasas de expectro extendido): en el fenotipo se encuentra resistencia a cefalosporinas de tercera y cuarta generación y son inhibidas por ácido clavulánico. Las pruebas fenotípicas para su detección se basan en esta capacidad inhibitoria del A. clavulánico como el E-test. También pueden usarse complementariamente pruebas de EDTA y ácido borónico para la diferenciación con metalo-betalactamasas $(13,14)$.

- Amp C (Beta lactamasas de la clase C de Ambler): el fenotipo se caracteriza por resistencia a penicilinas, beta lactámicos con inhibidores de beta-lactamasa, cefalosporinas de primera y segunda generación, así como las de tercera generación en grado variable. Los marcadores de mayor sensibilidad son la sensibilidad intermedia o resistencia a amoxicilina - ácido clavulánico y algunas cefalosporinas de tercera generación. Un marcador fenotípico usado para diferenciar AmpC de BLEE es la cefoxitina, donde los aislamientos con fenotipo AmpC son resistentes a cefoxitina mientras que los BLEE son sensibles. Cabe resaltar que el género Salmonella presenta resistencia intrínseca al cefoxitin $(13,14)$.

- ASSut: Se caracteriza por la presencia de resistencia a ampicilina, estreptomicina, sulfonamidas y tetraciclina (15).

Dentro de las variables de caracterización demográfica se incluye el estrato socioeconómico, que corresponde a valores de 1 a 6 que se utilizan para la clasificación de las viviendas en Colombia. Para la jerarquización se incluyen aspectos de construcción de la vivienda y entorno: en este rango de valores existe mayor bienestar de forma ascendente en las condiciones de la vivienda, las cuales usualmente son proporcionales a características de salud, educación y solvencia económica.

Todos los análisis fueron realizados utilizando el software estadístico Stata 13® (StataCorp, CollegeStation, Texas, USA).

\section{Resultados}

Entre los aislamientos obtenidos de hemocultivos, coprocultivos y otras muestras huma-
nas se recolectaron en total 60 casos. En la tabla 1 se encuentran las características de la población. 
Al realizar la distribución por años, veinte casos correspondían al año 2015, veinticuatro al año 2016 y 16 al año 2017. La mediana de la edad fue de 56 años, con rangos entre 18 a 88 años (RIQ 45, 75- 70). $46 \%$ eran de sexo femenino y $54 \%$ de sexo masculino.

Tabla 1. Características de la población infectada por Salmonella SP.

\begin{tabular}{|c|c|}
\hline Variable & $\begin{array}{c}\text { Número (\%) } \\
n=60\end{array}$ \\
\hline \multicolumn{2}{|c|}{ Características de la población } \\
\hline Femenino & $28(46.6)$ \\
\hline Masculino & $32(53.3)$ \\
\hline Casos 2014 & $16(25)$ \\
\hline Casos 2015 & $24(40)$ \\
\hline Casos 2016 & $20(33)$ \\
\hline Promedio de edad & 56.2 años \\
\hline Estrato 1 & $5(8.3)$ \\
\hline Estrato 2 & $15(25)$ \\
\hline Estrato 3 & $25(41.6)$ \\
\hline Estrato 4 & $13(21.6)$ \\
\hline Estrato $5-6$ & $1(1.6)$ \\
\hline Infección adquirida en la comunidad & 47 (78.3) \\
\hline Infección adquirida en el hospital & $13(21.6)$ \\
\hline \multicolumn{2}{|c|}{$\begin{array}{l}\text { Comorbilidades } \\
\end{array}$} \\
\hline Hipertensión arterial & $15(25)$ \\
\hline Enfermedad autoinmune & $12(20)$ \\
\hline Diabetes mellitus & $11(18.3)$ \\
\hline Falla cardíaca & $10(16.6)$ \\
\hline Colelitiasis & $10(16.6)$ \\
\hline VIH & $7(11.6)$ \\
\hline Enfermedad renal crónica & 7 (11.6) \\
\hline Hipotiroidismo & $6(10)$ \\
\hline \multicolumn{2}{|c|}{ Hallazgos de laboratorio } \\
\hline Leucocitos (mediana) & $8250 \mathrm{cel} / \mathrm{ul}$ \\
\hline Neutrófilos (mediana) & $6350 \mathrm{cel} / \mathrm{ul}$ \\
\hline Plaquetas (mediana) & $224000 \mathrm{cel} / \mathrm{ul}$ \\
\hline Hemoglobina (mediana) & $12 \mathrm{gr} / \mathrm{dl}$ \\
\hline \multicolumn{2}{|c|}{$\begin{array}{r}\text { Perfil clínico } \\
\end{array}$} \\
\hline Bacteriemia & $26(43)$ \\
\hline Gastroenteritis & $14(23)$ \\
\hline Fiebre tifoidea & $11(18.3)$ \\
\hline Infección órgano & $9(15)$ \\
\hline Bacteriuria asintomática & $2(3.3)$ \\
\hline Cirrosis & $1(1.6)$ \\
\hline
\end{tabular}


La mayor frecuencia se encontró en pacientes provenientes de estrato 3, con 25 casos (41.6\%), seguido de estrato 2 con 15 casos (25\%) y estrato 4 con 13 casos (21.6\%). El $78 \%$ de las infecciones fueron adquiridas en la comunidad y el $21.6 \%$ adquiridas en el hospital según las definiciones de infecciones asociadas al cuidado de la salud dadas por la cDc(16).

Las comorbilidades más frecuentes fueron hipertensión arterial (25\%), enfermedad autoinmune ( $20 \%$ ), diabetes mellitus (18.3\%) y falla cardíaca (16.6\%), el $11.6 \%$ tenían diagnóstico de vir y el $16.6 \%$ tenían colelitiasis. Dentro del grupo de enfermedades autoinmunes se encontraron con mayor frecuencia lupus eritematoso sistémico y artritis reumatoide.

Con relación al tipo de muestra, la mayor parte fueron hemocultivos en un $53 \%$, seguido de coprocultivos, $30 \%$, y otras secreciones $25 \%$ (tabla 2 ).

Tabla 2. Procedencia del aislamiento y perfil de resistencia

\begin{tabular}{cc} 
Variable & $\begin{array}{c}\text { Número (\%) } \\
\mathbf{n = 6 0}\end{array}$ \\
\hline \multicolumn{1}{c}{ Método de aislamiento } \\
\hline Hemocultivo & $32(53)$ \\
Coprocultivo & $18(30)$ \\
Otra secreción & $15(25)$ \\
\hline \multicolumn{1}{c}{ Perfil de resistencia } & \\
\hline BLEE & 0 \\
ASSut & 0 \\
Fluoroquinolonas & 0 \\
Amp C & $1(1.6)$ \\
Ampicilina Sulbactam & $1(1.6)$ \\
\hline
\end{tabular}

Abreviaturas: BLEE: Betalactamasas de espectro extendido ASSut: Resistencia a ampicilina, estreptomicina, sulfonamidas y tetraciclina, Amp C: Beta lactamasas de la clase C de Ambler.

Se encontró un caso de Salmonella typhi. Una de las limitaciones del estudio fue determinar el serotipo más frecuente, dado que la serotipificación no es realizada por el sistema automatizado, por lo que los serotipos de Salmonella diferente a S. typhise denominan como Salmonella group.

Los fenotipos de resistencia encontrados fueron Amp c en un caso y no se encontraron casos que cumplieran los criterios de BLEE, Assut o casos de resistencia a flouroquinolonas. Respecto a la susceptibilidad por antibióticos solo se encontró un aislamiento con resistencia a ampicilina, uno a ampicilina sulbactam, sin resistencia a trimetoprimcotrimoxazol ni ceftriaxona (tabla 2).

Dentro de la presentación clínica, el $43 \%$ correspondía a bacteriemia, $23 \%$ a gastroenteritis, $18.3 \%$ a fiebre tifoidea, $15 \%$ a infección de órgano y $2 \%$ a bacteriuria asintomática. La complicación más frecuente fue neumonía (5\%), seguida de mediastinitis, aneurima 
micótico, pielonefritis, endocarditis infecciosa y absceso hepático (tabla 3). La mortalidad intrahospitalaria fue del $15 \%(n=60)$ sin poder determinar el porcentaje de muerte asociada al tipo de infección.

Tabla 3. Complicaciones de las infecciones asociadas a Salmonella SP

\begin{tabular}{cc}
\hline Variable & $\begin{array}{c}\text { Número (\%) } \\
\mathbf{n}=\mathbf{6 0}\end{array}$ \\
\hline Complicaciones asociadas a la infección por Salmonella sp \\
\hline Neumonía & $3(5)$ \\
Mediastinitis & $1(1.6)$ \\
Aneurisma micótico & $1(1.6)$ \\
Pielonefritis & $1(1.6)$ \\
Endocarditis infecciosa & $1(1.6)$ \\
Absceso hepático & $1(1.6)$ \\
\hline
\end{tabular}

Teniendo en cuenta que solo se tuvieron tres casos de perfil de susceptibilidad diferente al usual no fue posible plantear una asociación entre el perfil de resistencia y otras variables estudiadas (estrato socioeconómico, comorbilidades, síndrome de presentación clínica).

\section{Discusión}

$\mathrm{E}$

xisten tres estudios en los que se analizó el perfil de resistencia de muestras procedentes de humanos. El estudio más grande realizado al momento incluyó 2092 pacientes procedentes de Nepal entre los años 2005 a 2014 con diagnóstico de fiebre entérica (17). El número de pacientes fue aleatorizado a varios brazos de antibióticos para evaluar la respuesta a los mismos (gatifloxacina, cefixime, cloranfenicol, ofloxacina, ceftriaxona). Dentro de los aislamientos en estos pacientes, 581 correspondieron a $S$. typhi y 274 a $S$. paratyphi. Se encontraron similares tasas de falla a diferencia de los pacientes, por lo que es importante recalcar que aquellos pacientes con cultivos negativos tuvieron más probabilidad de fallar a esquemas como cefixime, ceftriaxona y cloranfenicol comparado con quinolona. Aunque no se reporta el porcentaje de resistencia en el total de los aislamientos, se representa el aumento en la concentración mínima inhibitoria (MIc) de este grupo de antibióticos a lo largo del período nombrado. Se reporta un aumento en la concentración mínima inhibitoria (мгс) de quinolonas, ácido nalidíxico, ofloxacina, a lo largo del tiempo, con disminución en la mic para azitromicina y ceftriaxona, y sin aumento significativo en cloranfenicol y cotrimoxazol.

En Italia se realizó un estudio que analizó 734 aislamientos de Salmonella sp provenientes de especímenes humanos entre los años 2011 a 2012, dos aislamientos correspondieron 
a S. typhi, uno a Paratyphi B, y el $99 \%$ a serotipos no tifoideos, lo cual es comparable con nuestros datos (15). De las muestras del estudio en mención, el 38.4\% eran susceptibles a todos los antibióticos; $12.1 \%$ exhibieron resistencia a dos clases de antibióticos; $13.4 \%$ se clasificaron como multiresistentes (resistencia a tres o más clases de antibióticos no relacionados estructuralmente); $36.1 \%$ eran resistentes a ampicilina, estreptomicina, sulfonamidas y tetraciclinas (patrón de resistencia Assut). Los aislamientos resistentes a ciprofloxacina y ácido nalidíxico fueron $0.5 \%$. Solo tres aislamientos mostraron fenotipo BLEE o Amp C.

En el estudio más reciente, realizado del año 2015 al año 2016 de muestras provenientes de humanos en China se analizan 52 aislamientos (18). La resistencia a ampicilina se encontró en $57.6 \%$ de los aislamientos, seguido en frecuencia de resistencia a tetraciclinas (40.38\%), cotrimoxazole (15.3\%), azitromicina (11.5\%) y ciprofloxacina en (11.54\%).

Debido al bajo número de muestra y al tipo de estudio, no es posible extrapolar los resultados para establecer si hay un cambio en el perfil de susceptibilidad de Salmonella $s p$ en Colombia. Sin embargo, hasta el momento no hay otros estudios en el país que reporten la resistencia en aislamientos de este microorganismo en muestras de secreciones humanas. Como parte de la epidemiología de la Fundación cardioinfantil, se observa una disminución en la resistencia a ampicilina, cefalosporinas y quinolonas a partir del último reporte del Instituto Nacional de Salud (11).

Dado el bajo número de aislamientos con un patrón de susceptibilidad no usual (dos casos), no fue posible una asociación entre las características sociodemográficas o clínicas y los patrones de resistencia encontrados.

Partiendo de los resultados de este estudio, que muestran que la mayoría de los aislamientos persisten siendo sensibles a ampicilina, se sugiere realizar un estudio con una mayor muestra poblacional para determinar el perfil de resistencia de Salmonella sp en Colombia. De esta forma, su caracterización permitirá elegir otros antibióticos diferentes a quinolonas como base del tratamiento.

\section{Contribución de los autores}

Todos los autores participaron en la investigación y en la planeación del artículo, así como en el desarrollo y la aprobación final de esta versión.

\section{Financiación}

T a realización de estudio de Filmarray se realizó a discreción del medico tratante y se Uencuentra dentro de los estudios diagnósticos realizados en la Fundación Cardioinfantil. 


\section{Conflicto de intereses}

$\mathrm{N}^{\text {mesuroudedaratal }}$

\section{Referencias}

1. Haselbeck AH, Panzner U, Im J, Baker S, Meyer CG, Marks F. Current perspectives on invasive nontyphoidal Salmonella disease. Curr Opin Infect Dis. 2017;30(5):498-503. Doi: 10.1097/QCO.0000000000000398

2. Uche IV, MacLennan CA, Saul A. A Systematic review of the incidence, risk factors and case fatality rates of invasive nontyphoidal Salmonella (iNTS) Disease in Africa (1966 to 2014). PLoS Negl Trop Dis. 2017;11(1): 1-28. Doi: 10.1371/journal.pntd.0005118

3. Marks F, von Kalckreuth V, Aaby P, Adu-Sarkodie Y, El Tayeb MA, Ali M, et al. Incidence of invasive salmonella disease in sub-Saharan Africa: a multicentre population-based surveillance study. Lancet. Glob. Heal. 2017;5(3):310-23. Doi: 10.1016/S2214-109X(17)30022-0

4. Crump JA, Mintz ED. Global trends in typhoid and paratyphoid Fever. Clin. Infect. Dis. 2010;50(2):241-6. Doi: 10.1086/649541

5. Instituto Nacional de Salud de Colombia. Fiebre tifoidea y paratifoidea Colombia. [Internet] 2017 [Acceso 29 May 2019]. Disponible en: https://www.ins.gov.co/buscador-eventos/Informesdeevento/FIEBRE\%20TIFOIDEA\%20Y\%20PARATIFOIDEA\%20 2017.pdf

6. Ohn W, Hendriksen RS, Mikoleit ML, Keddy KH, RLO. Typhoid Fever. Lancet. 2015;385:1136-45. Doi: 10.1016/S0140-6736(13)62708-7

7. De Toro M, Seral C, Rojo-Bezares B, Torres C, Castillo FJ, Sáenz Y. Resistencia a antibióticos y factores de virulencia en aislados clínicos de Salmonella enterica ₹. Enferm Infecc Microbiol Clin. 2014;32(1):4-10. Doi: 10.1016/j.eimc.2013.03.006

8. Capalonga R, Ramos RC, Both JMC, Soeiro MLT, Longaray SM, Haas S, et al. Salmonella serotypes, resistance patterns, and food vehicles of salmonellosis in southern Brazil between 2007 and 2012. J Infect Dev Ctries. 2014;8(7):811-7. Doi: 10.3855/jidc.3791

9. Kuang D, Zhang J, Xu X, Shi W, Chen S, Yang X, et al. Emerging high-level cipro fl oxacin resistance and molecular basis of resistance in Salmonella enterica from humans, food and animals. Int J Food Microbiol. 2018;280:1-9. Doi: 10.1016/j.ijfoodmicro.2018.05.001

10. Medalla F, Gu W, Mahon BE, Judd M, Folster J, Griffin PM, et al. Estimated Incidence of Antimicrobial Drug-Resistant Nontyphoidal Salmonella Infections, United States, 20042012. Emerg Infect Dis. 2016;23(1):29-37. Doi: 10.3201/eid2301.160771

11. Instituto Nacional de Salud de Colombia. Características de los aislamientos de Salmonella spp en Colombia. Resultados de la vigilancia 2003 - 2013. [Internet] 2013 [Acceso 29 May 2019]. Disponible en: https://www.ins.gov.co/buscador-eventos/Informacin $\% 20 \mathrm{de} \% 20$ laboratorio/Informe\%20Vigilancia\%20por\%20laboratorio\%20de\%20Salmonella $\% 20$ spp\%202000-2013.pdf 
12. Wayne, PA. Clinical and laboratory standards institute. Performance Standards for Antimicrobial Susceptibility Testing. 29a edición. CLSI supplement M100. CLSI, 2019.

13. Vasoo S, Mrcp M, Pharmd JNB, Tosh PK. Emerging issues in gram-negative Bacterial resistance. Mayo Clin Proc [Internet]. 2015;90(3):395-403. Doi: 10.1016/j.mayocp.2014.12.002

14. Navarro F, Calvo J, Cantón R, Fernández-cuenca F. Detección fenotípica de mecanismos de resistencia en microorganismos gramnegativos. Enferm Infecc Microbiol Clin. 2011;29(7):524-34.

15. Frasson I, Bettanello S, De Canale E, Richter SN, Palu G. Serotype epidemiology and multidrug resistance patterns of Salmonella enterica infecting humans in Italy. Gut Pathog. 2016;8:26. Doi: 10.1186/s13099-016-0110-8

16. Horan TC, Andrus M, Dudeck MA. CDC/NHSN surveillance definition of health care-associated infection and criteria for specific types of infections in the acute care setting. Am J Infect Control. 2008;36(5):309-32.

17. Thompson CN, Karkey A, Dongol S, Arjyal A, Wolbers M, Darton T, et al. Treatment response in enteric fever in an era of increasing antimicrobial resistance: an individual patient data analysis of 2092 participants enrolled into 4 randomized, controlled trials in Nepal. Clin Infect Dis. 2017;64(11):1522-31. Doi: 10.1093/cid/cix185

18. Luo Y, Yi W, Yao Y, Zhu N, Qin P. Characteristic diversity and antimicrobial resistance of Salmonella from gastroenteritis. J Infect Chemother [Internet]. 2018;24(4):251-5. Doi: 10.1016/j.jiac.2017.11.003 